\title{
Stress-strain state of a soil array with a cylindrical cavity supported by a reinforcing ring
}

\author{
Vladimir Andreev* and Vladislav Nosyrin \\ Moscow State University of Civil Engineering, Yaroslavskoe shosse, 26, Moscow, 129337, Russia
}

\begin{abstract}
The article deals with the problem of calculating a stress-strain soil massif with a horizontal cylindrical cavity created by an explosion (for example, drilling and blasting method of penetration), in the presence of a reinforcing ring. The calculation takes into account the radial local inhomogeneity of the array near the cavity, due to the explosive effects. The problem is solved in a two-dimensional formulation (flat deformed state). The solution uses a numerical-analytical method that reduces the solution to a system of ordinary differential equations with variable coefficients.
\end{abstract}

\section{Introduction}

In [1], a numerical-analytical method is presented for solving the plane problem of elasticity theory in polar coordinates for radially inhomogeneous bodies. This method can be used for many practical problems, including the problem considered in this paper. As shown in $[2,3]$, in the rock during an explosion, a change in the modulus of deformation $E$ occurs. Depending on the structure of the soil or rock, or compaction occurs $[3,4]$, which leads to an increase in strength and deformation characteristics in the area of the array surrounding the hole, or loosening or the formation of fractured rock $[5,6]$ with a corresponding decrease in the listed characteristics in the near zone of the explosion. In order to strengthen underground cavities, including those created with the help of an explosion, various techniques are used - installation of reinforcing rings made of cast iron, concrete or reinforced concrete, freezing of the massif, gunning with concrete, etc.

In the calculation below, it was assumed that the change in the elastic modulus of a soil massif is described by the formula $[7,8]$ :

$$
E_{2}(r)=E_{20}\left[1+\left(k_{2}-1\right)\left(\frac{a}{r}\right)^{m_{2}}\right] .
$$

This function can well approximate experimental curves. By choosing the appropriate $m$, it is possible to describe dependencies with both slow and fast rates of convergence $E$ to the value $E_{0}$, which corresponds to a larger or smaller zone of the array with modified

* Corresponding author: asv@mgsu.ru 
properties. If $k>1$, relation (1) corresponds to compaction of the rock, and at $k<1$ its loosening.

In fig. 1 shows schematically the dependences of described by this formula for various values of the coefficients $k_{2}$ and $m_{2}$.

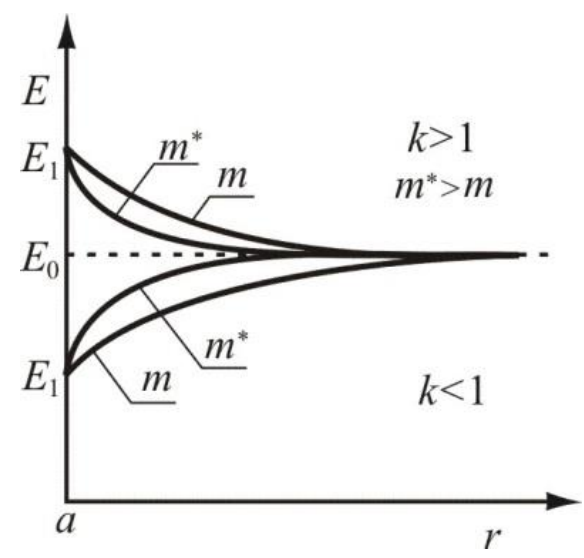

Fig.1. The change of the elastic modulus in the ground

The properties of the reinforcing ring, depending on the technology of its manufacture, may be different. For example, if the ring is made of a homogeneous material, then $E_{1}=$ const $=E_{10}$. When freezing or gunning occurs, the array is unevenly radially reinforced, and in this case, the function can be used to describe the change in the modulus of elasticity in the ring:

$$
E_{1}(r)=E_{10}\left(\frac{r}{a}\right)^{\delta_{1}}
$$

In formulas (1) and (2), the number 1 corresponds to the reinforcing ring, and 2 - to the soil massif, the number 0 corresponds to the initial (undisturbed) material of the ring and the massif.

In connection with the absence or insufficient amount of experimental data on changes in the Poisson's ratio $v$ of the reinforcing ring and the array, they were considered constant.

\section{Statement of the problem}

In fig. 2 shows the design scheme of the problem in which $a$ is the radius of the cylindrical cavity and the inner radius of the ring, $c$ is the outer radius of the ring, $b$ is the radius of the cut out part of the array, $H$ is the depth of the cavity, $\gamma$ is the specific gravity of the soil, $v_{2}$ - Poisson's ratio of the soil.

The cavity and ring are stress concentrators. As is well known, in a plane problem in polar coordinates, the stresses decrease inversely proportional to the square of the radius, which allows you to choose a radius $b 5$ to 10 times larger than $a$. Assuming that $H>>b$, then the vertical pressure of the soil at a depth of $H-b$ and $H+b$ can be considered approximately the same. In the horizontal direction, the pressure lateral repulse acts.

The problem is solved in displacements in polar coordinates. We write the basic equations: 


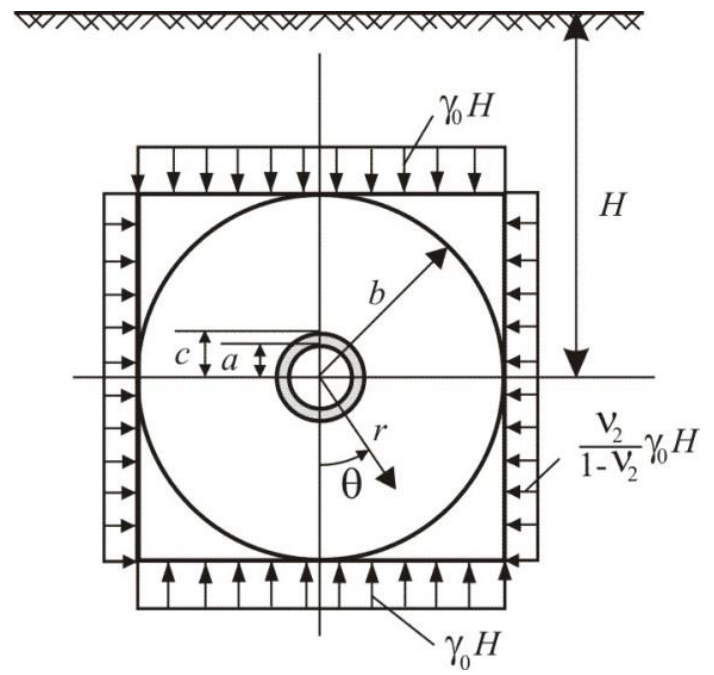

Fig. 2. The design scheme of an array with a cylindrical cavity supported by the reinforcing ring

Hooke's law in the form of Lame

$$
\begin{gathered}
\sigma_{r}=\lambda(r)\left(\frac{\partial u}{\partial r}+\frac{u}{r}+\frac{1}{r} \frac{\partial v}{\partial \theta}\right)+2 \mu(r) \frac{\partial u}{\partial r} \\
\sigma_{\theta}=\lambda(r)\left(\frac{\partial u}{\partial r}+\frac{u}{r}+\frac{1}{r} \frac{\partial v}{\partial \theta}\right)+2 \mu(r)\left(\frac{u}{r}+\frac{1}{r} \frac{\partial v}{\partial \theta}\right) \\
\tau_{r \theta}=\mu(r)\left[r \frac{\partial}{\partial r}\left(\frac{v}{r}\right)+\frac{1}{r} \frac{\partial u}{\partial \theta}\right] .
\end{gathered}
$$

Equilibrium equations

$$
\frac{\partial \sigma_{r}}{\partial r}+\frac{1}{r} \frac{\partial \tau_{r \theta}}{\partial \theta}+\frac{\sigma_{r}-\sigma_{\theta}}{r}=0 ; \quad \frac{\partial \tau_{r \theta}}{\partial r}+\frac{1}{r} \frac{\partial \sigma_{\theta}}{\partial \theta}+\frac{2 \tau_{r \theta}}{r}=0
$$

Substituting (3) into (4), we obtain a system of two partial differential equations [8]:

$$
\begin{aligned}
& (\lambda+2 \mu)\left(\frac{\partial^{2} u}{\partial r^{2}}+\frac{1}{r} \frac{\partial u}{\partial r}-\frac{u}{r^{2}}\right)+\frac{\mu}{r^{2}} \frac{\partial^{2} u}{\partial \theta^{2}}+\frac{\lambda+\mu}{r} \frac{\partial^{2} v}{\partial r \partial \theta}-\frac{\lambda+3 \mu}{r^{2}} \frac{\partial v}{\partial \theta}+ \\
& +\frac{\partial(\lambda+2 \mu)}{\partial r} \frac{\partial u}{\partial r}+\frac{1}{r} \frac{\partial \lambda}{\partial r}\left(u+\frac{\partial v}{\partial \theta}\right)=0 \\
& \mu\left(\frac{\partial^{2} v}{\partial r^{2}}+\frac{1}{r} \frac{\partial v}{\partial r}-\frac{v}{r^{2}}\right)+\frac{\lambda+\mu}{r} \frac{\partial^{2} u}{\partial r \partial \theta}+\frac{\lambda+3 \mu}{r^{2}} \frac{\partial u}{\partial \theta}+\frac{\lambda+2 \mu}{r^{2}} \frac{\partial^{2} v}{\partial \theta^{2}}+ \\
& +\frac{1}{r} \frac{\partial \mu}{\partial r}\left(\frac{\partial u}{\partial \theta}+r \frac{\partial v}{\partial r}-v\right)=0
\end{aligned}
$$

Here $\lambda=\lambda(r)$ and $\mu=\mu(r)$. 


\section{Method of solution}

To solve equations (5), the method of separation of variables [1,9] is used, which is the development of a generalized solution of the plane problem in polar coordinates of $\mathrm{J}$. $\mathrm{H}$. Michell $[10,11]$ to the case for radially inhomogeneous bodies. If the solution of J. H. Michell is constructed for the stress function, then the solution form proposed below is written for displacements [1]:

$$
\begin{aligned}
\left(\begin{array}{l}
u \\
v
\end{array}\right)= & \left(\begin{array}{l}
\varphi_{0} \\
\psi_{0}
\end{array}\right)+\left(\begin{array}{l}
\varphi_{1} \\
\psi_{1}
\end{array}\right) \cdot \theta+\left(\begin{array}{c}
\varphi_{2} \\
\psi_{2}
\end{array}\right) \cdot \theta \sin \theta+\left(\begin{array}{c}
\varphi_{3} \\
\psi_{3}
\end{array}\right) \cdot \theta \cos \theta+\left(\begin{array}{c}
\varphi_{4} \\
\psi_{4}
\end{array}\right) \cdot \sin \theta+\left(\begin{array}{c}
\varphi_{5} \\
\psi_{5}
\end{array}\right) \cdot \cos \theta+ \\
& +\sum_{n=2}^{\infty}\left[\left(\begin{array}{c}
\varphi_{s n} \\
\psi_{s n}
\end{array}\right) \sin n \theta+\left(\begin{array}{c}
\varphi_{c n} \\
\psi_{c n}
\end{array}\right) \cos n \theta\right],
\end{aligned}
$$

where $\varphi_{0}, \ldots . \psi_{c n}$ depend only on the radius. The upper functions in parentheses correspond to the displacements $u$, the lower $-v$, the indices $s$ and $c$ correspond to the functions multiplied by the sine and cosine of the angular parameter.

Substituting (6) into (5) leads to an infinite, partially decomposing system of ordinary differential equations with variable coefficients with respect to the functions $\varphi_{i}(r)$ и $\psi_{i}(r)$.

The boundary conditions in the problem are written as follows:

$$
\begin{array}{ll}
r=a, & \sigma_{r, 1}=\tau_{r \theta, 1}=0 \\
r=c, & \sigma_{r, 1}=\sigma_{r, 2} ; \tau_{r \theta, 1}=\tau_{r \theta, 2} ; \\
& u_{1}=u_{2} ; v_{1}=v_{2} ; \\
r=b, & \sigma_{r, 2}=-\frac{\gamma_{0} H}{2(1-v)}-\frac{1-2 v}{2(1-v)} \gamma_{0} H \cos 2 \theta \\
& \tau_{r \theta, 2}=\frac{1-2 v}{2(1-v)} \gamma_{0} H \sin 2 \theta .
\end{array}
$$

Here it is assumed that on the border of two layers $(r=c)$ conditions of ideal contact are fulfilled. The loads entering the conditions at the boundary $r=b$ can be obtained from the vertical $\left(\gamma_{0} H\right)$ and horizontal $\left(\gamma_{0} H \frac{v_{2}}{1-v_{2}}\right)$ pressures (see Fig. 2) by changing the variables.

Obviously, in order to satisfy conditions (5) in solutions for both layers $(j=1,2)$ in general expressions (4), it suffices to confine to terms containing functions $\varphi_{0}^{(j)}, \varphi_{c 2}^{(j)}$ and $\psi_{s 2}^{(j)}$. Substituting these functions in (5) we obtain the following equations:

$$
\begin{gathered}
(\lambda+2 \mu)\left(\varphi_{0}^{\prime \prime}+\frac{\varphi_{0}^{\prime}}{r}-\frac{\varphi_{0}}{r^{2}}\right)+\frac{\lambda+\mu}{r} \psi_{1}^{\prime}-\frac{\lambda+3 \mu}{r^{2}} \psi_{1}+\left(\lambda^{\prime}+2 \mu^{\prime}\right) \varphi_{0}^{\prime}+\frac{\lambda^{\prime}}{r}\left(\varphi_{0}+\psi_{1}\right)=0 \\
-2 \frac{\lambda+\mu}{r} \varphi_{c 2}^{\prime}-2 \frac{\lambda+3 \mu}{r^{2}} \varphi_{c 2}+\mu\left(\psi_{s 2}^{\prime \prime}+\frac{\psi_{s 2}^{\prime}}{r}-\frac{\psi_{s 2}}{r^{2}}\right)-4 \frac{\lambda+2 \mu}{r^{2}} \psi_{s 2}- \\
-\frac{\mu^{\prime}}{r}\left(2 \varphi_{c 2}-r \psi_{s 2}^{\prime}+\psi_{s 2}\right)=0
\end{gathered}
$$




$$
\begin{aligned}
& 2 \frac{\lambda+\mu}{r} \varphi_{s 2}^{\prime}+2 \frac{\lambda+3 \mu}{r^{2}} \varphi_{s 2}+\mu\left(\psi_{c 2}^{\prime \prime}+\frac{\psi_{c 2}^{\prime}}{r}-\frac{\psi_{c 2}}{r^{2}}\right)-4 \frac{\lambda+2 \mu}{r^{2}} \psi_{c 2}+ \\
& +\frac{\mu^{\prime}}{r}\left(2 \varphi_{s 2}+r \psi_{c 2}^{\prime}-\psi_{c 2}\right)=0 .
\end{aligned}
$$

These function $\varphi_{0}^{(j)}$ is defined from (8) at $\psi_{1}^{(j)}=0$ and functions $\varphi_{c 2}^{(j)}, \psi_{s 2}^{(j)}$ from (9) and (10). Equation (6) is reduced to a system of two first order equations, and equations (7) and (8) to a system of four first order equations. These systems of equations together with the boundary conditions (7) were solved in the complex Maple.

\section{Results and discussion}

As an example in fig. 3 and 4 shows the calculation results obtained with the following initial data: $\quad b / a=11 ; \quad c / a=1.2 ; \quad v_{1}=0.2 ; \quad v_{2}=0.15 ; \quad E_{10}=$ const $=2.5 \cdot 10^{4} \mathrm{MPa}$; $E_{20}=2 \cdot 10^{4} \mathrm{MPa} ; m_{2}=2$. The parameter $k_{2}$ included in (1) varied.

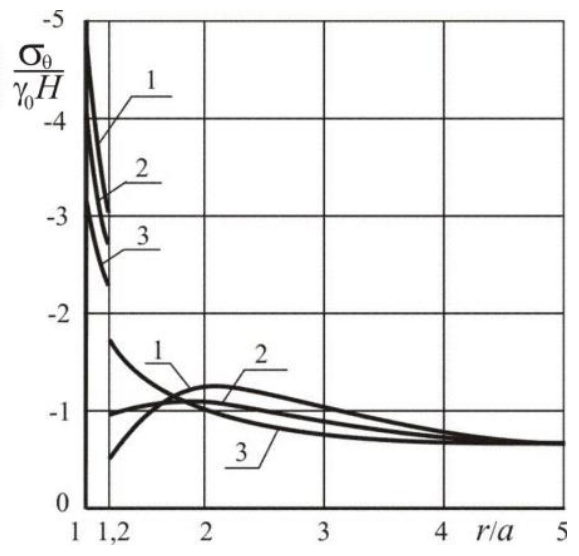

Fig. 3. Stresses $\sigma_{\theta}\left(\theta=90^{\circ}\right)$ in a inhomogeneous array with fastening ring

$1-k_{2}=0.2 ; 2-k_{2}=0.5 ; 3-k_{2}=1$.

The results indicate that with an increase in the degree of heterogeneity (the parameter $k_{2}$ decreases), the stresses in the reinforcing ring increase, which is quite natural if we take into accounting the equilibrium condition

$$
\int_{a}^{b} \sigma_{\theta} d r=\text { const. }
$$

The greatest stresses both in the ring and in the array occur at $\theta=90^{\circ}$ (see fig. 2). This fact is consistent with the solution of the Kirsch problem, since the pressure in the vertical direction is greater than in the horizontal. The last fact to pay attention to is to reduce the stresses to almost zero at the poles of the hole $\left(\theta=0,180^{\circ}\right)$. Calculations show that in some cases the stresses at these points can be tensile, which can lead to destruction, for example, if the reinforcing ring is made of concrete or the hole is strengthened by cementation. 

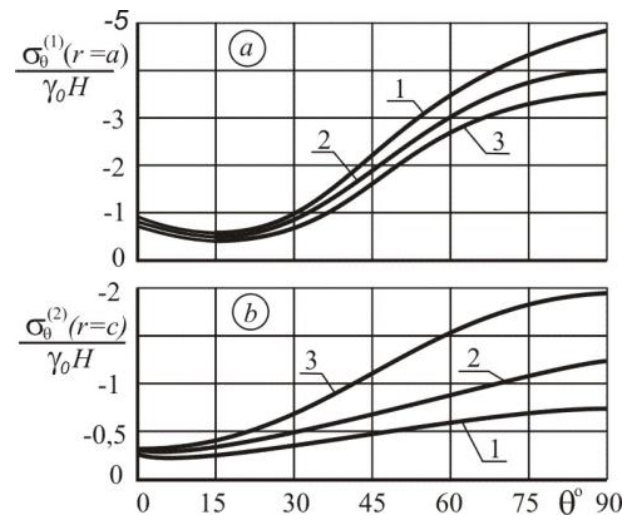

Fig. 4. The change of the greatest stress in the ring (a) and in the array (b) along the angular coordinate. Designations are the same as in fig. 3

This work was financially supported by the Ministry of Russian Education 7.6163.2017/6.7

\section{Conclusions}

The above solution in the article practically important problem illustrates the method for calculating the $[1,9]$ plane problem in polar coordinates for radially uniform bodies. Just as Michell generalized solution allows to obtain virtually any solution for the problem in the plane polar coordinates for a homogeneous material, discussed method allows to obtain a solution for the problem flat radially heterogeneous solids. As examples of the solutions of problems in various fields of technology, has been published in [14 - 19].

This work was financially supported by the Ministry of Education and Science (state task $\#$ 7.1524.2017/4.6).

\section{References}

1. V. I. Andreev, N. Yu.Tsybin, WIT Trans. on Model. and Simul., 57, 215 (2014)

2. K. Myasnikov, E. Leonov and N. Romadin, Compil. Peac. Nucl. Explos., III 1979 (1974)

3. A. Bakirov and other, Comparison of creep in free polymer rod and creep in polymer layer of the layered composite (Moscow, Nedra, 1981)

4. A.E. Babinets, Proc. of the Ing. Geol. Meet. prop. of rocks and meth. of their study, 1177 (1956)

5. B. N. Kutuzov, V.M. Globa and other Physical and mechanical properties of rocks in the near zone of the explosion in potash ores, Izv. universities. Mining Journal $10 \mathrm{pp}$ 87-91 (1974)

6. D. Rawson, P. Randolf and other, J. of Geophysical Research, 71143415 (1966)

7. I.V. Baklashov, B.A. Kartoziya, Rocks mechanics, (Moscow, Nedra, 1972)

8. V.I. Andreev, Mechanics of inhomogeneous bodies (Moscow, YURAIT, 2015)

9. V.I. Andreev, Appl. Mech. (KIEV) 23416 (1987)

10. J.H. Michell, Proc. London Math. Soc. 31100 (1899)

11. S.P. Timoshenko, J. Goodier Theory of elasticity (Moscow, Nauka, 1979)

12. S.K. Godunov, J. Comp. Math. and Phys. 26972 (1962) 
13. A.A. Samarsky, E.S. Nikolaev, Methods for solving grid equations (Moscow, Nauka, 1978)

14. A.S. Chepurnenko, B.M. Yazyev, , Adv. Mat. Res. 887-888 869 (2014)

15. V.I. Andreev, Adv. Mat. Res. 919-921 740 (2014)

16. A.S. Avershyev, V.I. Andreev, Appl. Mech. and Mater. 580-583 2974 (2014)

17. V.I. Andreev, Adv. in Eng. Res. (AER), 102, 232 (2017)

18. V.I. Andreev, E3S Web of Conf. 33, 02027 (2018)

19. V.I. Andreev. A.S. Avershyev, Educ. Res. 9, 458 (2018) 